\title{
ON ALMOST EVERYWHERE CONVERGENCE OF BOCHNER-RIESZ MEANS IN HIGHER DIMENSIONS
}

\author{
MICHAEL CHRIST
}

\begin{abstract}
In $\mathbf{R}^{n}$ define $\left(T_{\lambda, r} f\right)^{-}(\xi)=\hat{f}(\xi)\left(1-\left|r^{-1} \xi^{2}\right|\right)_{+}^{\lambda}$. If $n \geq 3$, $\lambda>\frac{1}{2}(n-1) /(n+1)$ and $2 \leq p<2 n /(n-1-2 \lambda)$, then $\lim _{r \rightarrow \infty} T_{\lambda, r} f(x)=f(x)$ a.e. for all $f \in L^{p}\left(\mathbf{R}^{n}\right)$.
\end{abstract}

The Bochner-Riesz operators in $\mathbf{R}^{n}$ are defined as $\left(T_{\lambda} f\right)^{-}(\xi)=\hat{f}(\xi)\left(1-|\xi|^{2}\right)_{+}^{\lambda}$, and the associated maximal operators are

$$
T_{\lambda}^{*} f(x)=\sup _{r>0}\left|\left(\hat{f} \cdot\left(1-|r \xi|^{2}\right)_{+}^{\lambda}\right)^{\vee}\right|(x) .
$$

It is conjectured that, when $\lambda>0, T_{\lambda}$ is bounded on $L^{p}$ if and only if $p_{0}^{\prime}(\lambda)<$ $p<p_{0}(\lambda)$, where $p_{0}(\lambda)=2 n /(n-1-2 \lambda)$. That the restrictions on $p$ and $\lambda$ are necessary was shown by Herz [7]. Carleson and Sjölin [3] proved the conjecture in dimension two. Moreover, in $\mathbf{R}^{2}$ Carbery [1] has established boundedness of $T_{\lambda}^{*}$ on $L^{p}$, and hence almost everywhere convergence of Bochner-Riesz means, for the same range of $p$ and $\lambda$ except for the added restriction $p \geq 2$. In dimensions greater than two it is known by work of C. Fefferman, Stein and Tomas $[\mathbf{5}, \mathbf{6}, \mathbf{1 0}]$ that $T_{\lambda}$ is bounded on $L^{p}$, provided $p_{0}^{\prime}(\lambda)<p<p_{0}(\lambda)$ and $\lambda>\frac{1}{2}(n-1) /(n+1)$, but the remaining cases have not been resolved. Our principal result is

THEOREM 1. $T_{\lambda}^{*}$ is bounded on $L^{p}\left(\mathbf{R}^{n}\right)$ whenever $2 \leq p<p_{0}(\lambda)$ and $\lambda>$ $\frac{1}{2}(n-1) /(n+1)$ for all $n \geq 3$.

Interest in $L^{p}$ bounds for $T_{\lambda}^{*}$ is due to the consequence then

Corollary. If $f \in L^{p}\left(\mathbf{R}^{n}\right), n \geq 3, \lambda>\frac{1}{2}(n-1) /(n+1)$ and $2 \leq p<p_{0}(\lambda)$,

$$
\lim _{r \rightarrow \infty}\left(\hat{f} \cdot\left(1-|\xi / r|^{2}\right)_{+}^{\lambda}\right)^{\smile}(x)=f(x) \quad \text { a.e. }
$$

The proof is based on the $L^{2}$ restriction theorem of Tomas and Stein [10]. Our second result is a small extension of that theorem, related in spirit to Theorem 1.

THEOREM 2. Suppose $\mu$ is a nonnegative radial measure on $\mathbf{R}^{n}$, satisfying $\mu(\{0\})=0$, and $n \geq 2$. Let $\gamma=n(n-1) /(n+1)$. Suppose $1<p \leq 2(n+1) /(n+3)$ and $q=((n-1) /(n+1)) p^{\prime}$. Then a necessary and sufficient condition that the weighted norm inequality $\|\hat{f}\|_{L^{q}\left(\mathbf{R}^{n}, d \mu\right)} \leq C\|f\|_{L^{p}\left(\mathbf{R}^{n}\right)}$ hold is that there exist $A<$ $\infty$ such that, for each $0<r<\infty, \mu\{r<|\xi|<2 r\} \leq A r^{\gamma}$.

Received by the editors August 9, 1984.

1980 Mathematics Subject Classification. Primary 42B15, 42B25. 
To begin the proof of Theorem 1 let us recall (Stein and Weiss [9] and Carbery [2]) that, for any $\varepsilon>0$,

$$
\left\|T_{\lambda}^{*} f\right\|_{p} \leq C\|M f\|_{p}+C(\varepsilon) \sum_{k=1}^{\infty} 2^{-k(\lambda-1 / 2-\varepsilon)}\left\|S_{2^{-k}} f\right\|_{p},
$$

where $M$ is the maximal operator of Hardy and Littlewood and each $S_{\delta}$ is a square function defined as follows:

Consider any $a \in C_{0}^{\infty}(\mathbf{R})$ supported in $\left[-\frac{1}{2}, \frac{1}{2}\right]$ and satisfying $\left\|D^{\alpha} a\right\|_{\infty} \leq 1$ for all $0 \leq \alpha \leq 10$. Let $\phi(\xi)=\phi^{(\delta)}(\xi)=a\left(\delta^{-1}(1-|\xi|)\right), \phi_{t}(\xi)=\phi(t \xi)$ and

$$
S_{\delta} f(x)=\left(\int_{0}^{\infty}\left|f * \check{\phi}_{t}\right|^{2}(x) t^{-1} d t\right)^{1 / 2} .
$$

(To be more precise, each $S_{2^{-k}}$ appearing in the above expression controlling $\left\|T_{\lambda}^{*} f\right\|_{p}$ is defined by means of an auxiliary function $a=a_{k}$ which depends on $k$.) Define $p_{0}=2(n+1) /(n-1)$ and $r=\left(\frac{1}{2} p_{0}\right)^{\prime}=(n+1) / 2$.

LEMMA 1. If $S_{\delta}$ is defined as above, then $\left\|S_{\delta} f\right\|_{p} \leq C(p) \delta^{1-n / 2 r}\|f\|_{p}$ for all $2 \leq p<p_{0}$.

By summing over all $\delta=2^{-k}, 0 \leq k$, we find (since $\left.\frac{1}{2}(n-1) /(n+1)=\frac{1}{2} n / r-\frac{1}{2}\right)$ that, if $2 \leq p<p_{0}$ and $\lambda>\frac{1}{2}(n-1) /(n+1)$, then $T_{\lambda}^{*}$ is bounded on $L^{p}$. The full conclusion of Theorem 1 may then be obtained by interpolating with the more elementary result: $T_{\lambda}^{*}$ is bounded on $L^{p}$, for all $1<p<\infty$, once $\lambda$ is greater than the critical index $\frac{1}{2}(n-1)$. (For in that case $T_{\lambda}^{*}$ is dominated pointwise by the Hardy-Littlewood maximal operator, since

$$
\left.\left|\left(\left(1-|\xi|^{2}\right)_{+}^{\lambda}\right)^{`}(x)\right| \leq C(1+|x|)^{-(n+1) / 2-\lambda} .\right)
$$

Because of work of Rubio de Francia [8] and Carbery [2] one expects that the square function $S_{\delta}$ should be controlled by a maximal operator. Let $M_{r} h=$ $M\left(h^{r}\right)^{1 / r}$ for any nonnegative function $h ; M$ always denotes the Hardy-Littlewood maximal operator.

LEMMA 2. For any $0 \leq h$ and $0<\delta \leq 1$,

$$
\int\left|S_{\delta} f\right|^{2}(x) h(x) d x \leq C \delta^{2-n / r} \int|f|^{2}(x) M_{r} h(x) d x .
$$

Lemma 1 is an immediate corollary. That the controlling operator $h \rightarrow M_{r} h$ should have such a simple form is unexpected; in dimension two the weighted inequality established by Carbery for the Bochner-Riesz multipliers involves averaging over rectangles with eccentricity $\delta^{-1 / 2}$ and arbitrary orientations.

Our proof relies on an argument given by Stein (see [6]), who reduces the problem of estimating the Bochner-Riesz operators to a local one on a fixed cube, and treats the local problem by applying the $L^{2}$ restriction theorem. For the local problem one has not only $L^{p_{0}}$ boundedness, but boundedness of the operator in question from $L^{2}$ to $L^{p_{0}}$. Our sole innovation is the observation that this stronger local information automatically carries with it a weighted inequality.

To formulate this principle abstractly we suppose that $\mathbf{R}^{n}$ has been partitioned into a regular lattice of cubes of sidelengths $2^{j}$, and that $T$ is a sublinear operator with the property that, whenever $f$ is supported in a cube $Q$ of the lattice, $T f$ is supported in a fixed dilate $Q^{*}$ of $Q$. 
LEMMA 3. Suppose $T$ is as above, $2<p_{0}$ and $r=\left(\frac{1}{2} p_{0}\right)^{\prime}$. Suppose that for any $Q,\|T f\|_{p_{0}} \leq A\|f\|_{2}$ for any $f$ supported in $Q$. Then for any $f$ defined on $\mathbf{R}^{n}$ and any testing function $h \geq 0$,

$$
\int|T F|^{2}(x) h(x) d x \leq C A^{2} 2^{j n / r} \int|f|^{2}(x) M_{r} h(x) d x .
$$

PrOOF. By the locality assumption it suffices to assume that $f$ is supported in one cube $Q$ of the lattice, and $h$ on $Q^{*}$. By Hölder's inequality

$$
\begin{aligned}
\int|T f|^{2} h & \leq\left(\int|T f|^{p_{0}}\right)^{2 / p_{0}}\left(\int_{Q^{*}} h^{r}\right)^{1 / r} \\
& \leq C A^{2}|Q|^{1 / r}\|f\|_{2}^{2}\left(\left|Q^{*}\right|^{-1} \int_{Q^{*}} h^{r}\right)^{1 / r} \\
& \leq C A^{2}|Q|^{1 / r}\|f\|_{2}^{2} \operatorname{Inf}_{x \in Q} M_{r} h(x) \\
& \leq C A^{2}|Q|^{1 / r} \int_{Q}|f|^{2} M_{r} h .
\end{aligned}
$$

To deduce Lemma 2, fix a cutoff function $\eta \in C_{0}^{\infty}\left(\mathbf{R}^{n}\right)$, identically one on $\{|x| \leq 1\}$ and supported on $\{|x| \leq 2\}$. Suppose $\delta>0$ is given, $2^{-i}>\delta \geq 2^{-i-1}$. Let $\zeta_{i}(x)=\eta\left(2^{-i} x\right)$, and $\zeta_{j}(x)=\eta\left(2^{-j} x\right)-\eta\left(2^{1-j} x\right)$ for all $j>i$.

Apply Lemma 3 to the vector-valued operator $T: L^{2} \rightarrow L^{P_{0}}\left(L^{2}[1 / 2,4]\right)$ defined by $T f(x, t)=\left(f *\left(\zeta_{j} \breve{\varphi}_{t}\right)\right)(x)$. Let $(\rho, \theta) \in \mathbf{R}^{+} \times S^{n-1}$ denote polar coordinates. Since $\zeta_{j}$ is a dilate of a fixed Schwartz function, $j \geq i$, and all moments of $\hat{\zeta}_{j}$ vanish when $j>i$, routine computation gives

$$
\left|\left(\hat{\zeta}_{j} * \varphi_{t}\right)(\rho, \theta)\right| \leq \begin{cases}C_{N} 2^{i-j}\left[1+2^{i}|\rho-t|\right]^{-N}, & \rho \in[1 / 4,8], \\ C_{N} 2^{i-j} \delta^{N}(1+\rho)^{-N}, & \text { otherwise }\end{cases}
$$

for any $N<\infty$ and any $t \in[1 / 2,4]$. Set $\hat{f}_{0}(\rho, \theta)=\hat{f}(\rho, \theta)$ for $\rho \in[1 / 4,8]$ and $\hat{f}_{0}(\rho, \theta)=0$ otherwise. Let $f_{1}=f-f_{0}$.

We may now show that the parameter $A^{2}$ of the lemma is at most $C \delta^{2} 4^{i-j}$. For any $f \in L^{2}$,

$$
\begin{aligned}
& \left\|\int_{1 / 2}^{4}|T f(x, t)|^{2} d t\right\|_{p_{0} / 2} \leq \int_{1 / 2}^{4}\left\|f * \zeta_{j} \check{\varphi}_{t}\right\|_{p_{0}}^{2} d t \\
& \quad \leq 2 \int_{1 / 2}^{4}\left\|f_{0} * \zeta_{j} \check{\varphi}_{t}\right\|_{p_{0}}^{2} d t+2 \int_{1 / 2}^{4}\left\|f_{1} * \zeta_{j} \check{\varphi}_{t}\right\|_{p_{0}}^{2} d t .
\end{aligned}
$$

The first term is the main one. It is

$$
\begin{aligned}
& 2 \int_{2^{-1}}^{4}\left\|\int_{2^{-2}}^{8} \int e^{i x \cdot \rho \theta} \hat{f}(\rho, \theta)\left(\hat{\zeta}_{j} * \varphi_{t}\right)(\rho, \theta) d \theta \rho^{n-1} d \rho\right\|_{p_{0}}^{2} d t \\
& \quad \leq C \int_{2^{-1}}^{4} \int_{2^{-2}}^{8}\left(\left\|\int e^{i x \cdot \rho \theta} \hat{f}(\rho, \theta)\left(\hat{\zeta}_{j} * \varphi_{t}\right)(\rho, \theta) d \theta\right\|_{p_{0}} d \rho\right)^{2} d t \\
& \quad \leq C \int_{2^{-1}}^{4} \int_{2^{-2}}^{8}\left\|\int e^{i x \cdot \rho \theta} \hat{f}(\rho, \theta)\left(\hat{\zeta}_{j} * \varphi_{t}\right)(\rho, \theta) d \theta\right\|_{p_{0}}^{2} d \rho\left[1+2^{i} \mid \rho-t \| B(t) d t\right.
\end{aligned}
$$


by the Cauchy-Schwarz inequality applied in the variable $\rho$, where $B(t)=$ $\int\left[1+2^{i}|\rho-t|^{-1} d \rho<C 2^{-i} \approx C \delta\right.$. Therefore combining the above bound for $\hat{\zeta}_{j} * \varphi_{t}$ with the $L^{2}$ restriction theorem of Tomas and Stein gives the bound

$$
\begin{aligned}
C \delta & \int_{2^{-1}}^{4} \int_{2^{-2}}^{8}\|\hat{f}(p, \cdot)\|_{L^{2}\left(S^{n-1}\right)}^{2} 2^{2(i-j)}\left[1+2^{i}|\rho-t|\right]^{1-2} d \rho d t \\
& \leq C \delta^{2} 4^{i-j} \int_{2^{-2}}^{8}\|\hat{f}(\rho, \cdot)\|_{L^{2}\left(S^{n-1}\right)}^{2} d \rho=C \delta^{2} 4^{i-j}\|f\|_{L^{2}}^{2} .
\end{aligned}
$$

A straightforward application of Plancherel's theorem and fractional integration, with no appeal to the restriction theorem, yields the same bound for the contribution of $f_{1}$.

Therefore by Lemma 3 ,

$$
\left\langle\int_{1 / 2}^{4}\left|f * \zeta_{j} \check{\varphi}_{t}\right|^{2} d t, h\right\rangle \leq C \delta^{2} 4^{i-j} 2^{j n / r}\left\langle|f|^{2}, M_{r} h\right\rangle .
$$

Now $n / r<2$, so summing the geometric series over all $j \geq i$ gives

$$
\left\langle\int_{1 / 2}^{4}\left|f * \check{\varphi}_{t}\right|^{2} d t, h\right\rangle \leq C \delta^{2-n / r}\left\langle|f|^{2}, M_{r} h\right\rangle .
$$

In order to conclude the proof via Littlewood-Paley theory we are forced to introduce one more collection of cutoff functions. Select $\rho \in C_{0}^{\infty}\left(\mathbf{R}^{n}\right)$, radial and supported in $\{1 \leq|\xi| \leq 3\}$ such that $\sum_{-\infty}^{\infty} \rho\left(2^{k} \xi\right) \equiv 1$ on $\mathbf{R}^{n} \backslash\{0\}$. $\rho_{k}$ denotes $\rho\left(2^{k} \cdot\right)$. Then

$$
\begin{aligned}
\int_{0}^{\infty}\left|f * \check{\phi}_{t}\right|^{2} t^{-1} d t & \leq 3 \sum_{k} \int_{0}^{\infty}\left|\left(f * \check{\rho}_{k}\right) * \check{\phi}_{t}\right|^{2} t^{-1} d t \\
& =3 \sum_{k} \int_{2^{k-1}}^{2^{k+2}}\left|\left(f * \check{\rho}_{k}\right) * \check{\phi}_{t}\right|^{2} t^{-1} d t
\end{aligned}
$$

By homogeneity and the case $\frac{1}{2} \leq t \leq 4$,

$$
\left\langle\int_{2^{k-1}}^{2^{k+2}}\left|f * \check{\rho}_{k} * \check{\phi}_{t}\right|^{2} t^{-1} d t, h\right\rangle \leq C \delta^{2-n / r}\left\langle\left|f * \rho_{k}\right|^{2}, M_{r} h\right\rangle
$$

and, therefore,

$$
\left\langle S_{\delta} f^{2}, h\right\rangle \leq C \delta^{2-n / r}\left\langle\sum_{k}\left|f * \check{\rho}_{k}\right|^{2}, M_{r} h\right\rangle \leq C \delta^{2-n / r}\left\langle|f|^{2}, M_{r} h\right\rangle .
$$

The last inequality follows from the weighted norm theory for singular integral operators, since $M_{r} h$ is an $A_{1}$ weight.

PROOF OF THEOREM 2. The necessity of the hypothesis follows from homogeneity considerations and the fact that $\mu\{1<|\xi|<2\}$ must be finite. Conversely, if $\mu$ satisfies the hypothesis, then it is an immediate corollary of the Tomas-Stein restriction theorem that

$$
\left(\int_{r}^{2 r}|\hat{f}(\xi)|^{q} d \mu(\xi)\right)^{1 / q} \leq C A\|f\|_{p} \quad \text { for any } 0<r<\infty .
$$


Let $f_{k}=\left(\hat{f} \rho_{k}\right)^{`}$ where $\left\{\rho_{k}\right\}$ are as above. Suppose $p=2(n+1) /(n+3)$, so $q=2$. Then

$$
\int|\hat{f}(\xi)|^{2} d \mu(\xi) \leq C \sum_{k} \int\left|\hat{f}_{k}(\xi)\right|^{2} d \mu(\xi) \leq C A^{2} \sum_{k}\left\|f_{k}\right\|_{p}^{2}
$$

But by Minkowski's inequality and the Littlewood-Paley theory,

$$
\sum_{k}\left\|f_{k}\right\|_{p}^{2} \leq\left\|\left(\sum_{k}\left|f_{k}\right|^{2}\right)^{1 / 2}\right\|_{p}^{2} \leq C\|f\|_{p}^{2}
$$

Since the case $p=1, q=\infty$ is trivial, the case $1<p<2(n+1) /(n+3)$ follows by interpolation.

REMARK. This argument is closely related to an almost-orthogonality lemma employed by the author in [4].

\section{REFERENCES}

1. A. Carbery, The boundedness of the maximal Bochner-Riesz operator on $L^{4}\left(\mathbf{R}^{2}\right)$, Duke Math. J. 50 (1983), 409-416.

2.,$A$ weighted inequality for the maximal Bochner-Riesz operator on $\mathbf{R}^{2}$, Trans. Amer. Math. Soc. 287 (1985), 673-680.

3. L. Carleson and P. Sjölin, Oscillatory integrals and a multiplier problem for the disc, Studia Math. 44 (1972), 287-299.

4. M. Christ, Convolution estimates for Cantor-Lebesgue measures, Preprint.

5. C. Fefferman, Inequalities for strongly singular convolution operators, Acta. Math. 124 (1970), 9-36.

6. __ A note on spherical summation multipliers, Israel J. Math. 15 (1973), 44-52.

7. C. Herz, On the mean inversion of Fourier and Hankel transforms, Proc. Nat. Acad. Sci. U.S.A. 40 (1954), 996-999.

8. J. L. Rubio de Francia, Weighted norm inequalities and vector valued inequalities, Proc. Conf. Harmonic Analysis, Minneapolis, 1981, Lecture Notes in Math., vol. 908, Springer-Verlag, Berlin and New York, 1982, pp. 86-101.

9. E. M. Stein and G. Weiss, Introduction to Fourier analysis on Euclidean spaces, Princeton Univ. Press, Princeton, N.J., 1971.

10. P. Tomas, Restriction theorems for the Fourier transform, Harmonic Analysis in Euclidean Space (G. Weiss and S. Wainger, Eds.), Proc. Sympos. Pure Math., Vol. 35, Part I, Amer. Math. Soc., Providence, R.I., 1979, pp. 111-114.

Department of Mathematics, Princeton University, Princeton, New Jersey 08544 